\title{
Quantized vortices in systems with pairing of spatially separated electrons and holes
}

\author{
S. I. Shevchenko \\ B. I. Verkin Institute for Low Temperature Physics and Engineering, \\ National Academy of Sciences of Ukraine, Kharkov, 310164, Ukraine \\ E-mail:shevchenko@ilt.kharkov.ua
}

Submitted September 10, 1996, revised March 3, 1997

\begin{abstract}
It is predicted that in systems with pairing of spatially separated electrons and holes the planar vortices, in which the electron-hole pairs rotate in the plane of the structure, can become energy-advantageous in a nonuniform magnetic field. In this case the vortices should form an ordered, though translation-noninvariant, structure.
\end{abstract}

PACS: $67.20 .+k$

The possibility of Bose condensation of real excitons, i.e., excitons whose size is smaller than the distance between them, was first pointed out in Ref. 1. Later, this subject was discussed in connection with exciton insulators [2], in which the excitons are large compared with the electron-electron distance. By analogy with He-II and superconductors, it was assumed that Bose condensation of excitons would lead to the appearance of superfluid properties in exciton system. Since an exciton is an electroneutral excitation, it cannot transfer either a charge or mass. There is, however, the possibility of a nondissipative transfer of energy and possibly of dipole and magnetic moments. This statement does not imply the true superfluidity. It only means that dissipation of the energy flow (or polarization) does not occur below the Bose condensation temperature during times shorter than the electron-hole recombination time $\tau_{r}$. It was shown later [3] that the interband transitions responsible for the value of $\tau_{r}$ lift the degeneracy of phase of the order parameter and cause a gap in the excitation spectrum, bringing the system from the superfluid state to the insulator state. Since it takes about $\tau_{r}$ for gap to form, the later statement is true for times longer than $\tau_{r}$. As a result, steady superfluidity in the exciton gas does not take place.

The problem of superfluidity of electron-hole gas was approached from a new standpoint in the publications [4,5], which called attention to the possibility of pairing of spatially separated electrons and holes (PSSEH). In systems with PSSEH the inter- band transitions coincide with the interlayer transitions and the probability of interlayer transitions can be easily changed by varying the thickness $d$ of a dielectric layer, which separates layers with electron and hole conductivities. Since the probability of interlayer transitions decreases exponentially with increasing $d$, and since the binding energy of electrons with holes falls off algebraically with $d$, the binding energy can reach $10^{2} \mathrm{~K}$, at $d \approx 10^{-6} \mathrm{~cm}$, but interband transitions can be completely disregarded. As a result, in systems with PSSEH, electron-hole pairs can go into a truly superfluid state. Moreover, in systems with PSSEH there is no local compensation of the electron current by a hole current, which makes it possible to observe supercurrents equal and opposite in direction, in layers with electron and hole conductivities. This means that in systems with PSSEH, a rather unusual superconductivity mechanism can be realized. In this connection, it was proposed to call superfluidity of electron-hole pairs in such systems «condenser superconductivity» [6].

The predictions made in Refs. 4-6 became of great interest when the progress in microelectronics resulted in the creation of the required structures. Many experimental [7-11] and theoretical [12-16] studies, in which the systems with PSSEH were analyzed, were published. However, in contrast to the $3 D$ systems, in which a considerable progress in obtaining degenerate gas of excitons, and perhaps Bose condensation of excitons [17] has recently been achieved, for systems with PSSEH it is im- 
possible at present to say with confidence that condenser superconductivity is realized under experimental conditions. Additional difficulties in recording of this superconductivity stem from the electrical neutrality of the pairs and the impossibility of using a traditional technique of measurement of the transport phenomena.

In the present paper we describe a new phenomenon in condenser superconductors, the observation of which is perhaps a more simple experimental problem than direct measurement of conductivity currents in electron and hole layers. We show that a nonuniform magnetic field can lead to the appearance of quantized vortices in the system in which electron-hole pairs rotate in the plane of the structure. We also show that although these vortices are very similar to the Onsager-Feynman and Abrikosov vortices, under certain conditions the structure formed by them differs radically from the structures formed by Onsager-Feynman and Abrikosov vortices.

Note that the behavior of the systems with PSSEH in a uniform field normal to a structure plane was considered many times. It was observed for the first time in Ref. 18, then in a series of studies [19], and quite recently in Refs. 20 and 21. In all those studies, the influence of a strong magnetic field $H$ on the pairing of electrons with holes was considered for the case in which the cyclotron radius $(c \hbar / e H)^{1 / 2}$ is lower than the Bohr radius $\varepsilon \hbar^{2} / m e^{2}$. The possibility of inducing planar vortices by a nonuniform magnetic field has not been considered until now. We consider here the case of weak magnetic fields $\left.\left[(c \hbar / e H)^{1 / 2}>>\varepsilon \hbar / m e^{2}\right)\right]$ in which the effect of the field on pairing can be ignored.

The system under consideration is a three-layer structure: two conducting two-dimensional layers are separated by a thin insulator layer. For definiteness, we assume that the lower layer has electron conductivity and the upper layer has hole conductivity. The Coulomb interaction leads to electronhole pairing, and the pairs form Bose gas which becomes superfluid below $T_{c}$ [6]. In the coherent phase the supercurrent is $\mathbf{j}_{e}=-e n_{s} \mathbf{v}_{s} \delta(z+d / 2)$ in the electron layer, and $\mathbf{j}_{h}=e n_{s} \mathbf{v}_{s} \delta(z-d / 2)$ in the hole layer. Here $n_{s}$ is the two-dimensional superfluid density and $-e$ is the electron charge. We assume that the conducting layers are infinitely thin, which leads to a $\delta$ function dependence of the current density on the $z$ coordinate. The superfluid velocity of the electron-hole pairs, in general, is [4]

$$
\mathbf{v}_{s}=\frac{\hbar}{m} \nabla \varphi-\frac{e}{m c}\left(\mathbf{A}_{h}-\mathbf{A}_{e}\right)
$$

Here $m$ is the pair mass; $\boldsymbol{\varphi}$ is the order parameter phase; $\mathbf{A}_{e}$ and $\mathbf{A}_{h}$ are the vector potentials in the electron and hole layers, respectively.

Here we assume that the thickness of the insulator layer, $d$, is small in comparison with the distance in which the vector potential $\mathbf{A}$ changes, so that the difference $\mathbf{A}_{h}-\mathbf{A}_{e}$ is

$$
\mathbf{A}_{h}-\mathbf{A}_{e}=\frac{\partial \mathbf{A}}{\partial z} d
$$

On the other hand, we consider $d$ large enough to ignore the tunnel transitions of the electrons between the conducting layers.

Our primary interest is in the velocity field of the planar vortex and the magnetic field created by the vortex. For the vortex, whose center is at $\rho=0$, the phase is

$$
\varphi=\arctan \frac{y}{x} .
$$

Because of the spatial separation of the electrons and holes, the rotation of the pairs generates a magnetic field in the surrounding space. To find the magnetic field, we must solve a Maxwell's equation

$$
\nabla \times \mathbf{H}=\frac{4 \pi}{c} \mathbf{j}(z) .
$$

Substituting the corresponding currents in the electron or hole layer instead of $\mathbf{j}$ in Eq. (4), writing $\mathbf{H}$ as $\mathbf{H}=\nabla \times \mathbf{A}$, and using the condition $\nabla \cdot \mathbf{A}=0$, we obtain

$$
-\Delta \mathbf{A}+\gamma\left(\frac{\partial \mathbf{A}}{\partial z}-\frac{\mathbf{S}}{d}\right)[\delta(z-d / 2)-\delta(z+d / 2)]=0 \text {. }
$$

Here the vector $\mathbf{S}=\hbar c \nabla \varphi / e$ has only the $\theta$ th component for the phase $\varphi$ from Eq. (3) and $S_{\theta}=\hbar c / e \rho$. We also introduce the dimensionless quantity $\gamma=4 \pi n_{s} e^{2} d / m c^{2}$.

To solve Eq. (5), we take the Fourier-transformation of the vector potential

$$
\mathbf{A}(\rho, z)=\int \mathbf{A}(\mathbf{q}, k) \exp \{i \mathbf{q} \boldsymbol{\rho}+i k z\} d^{2} q d k /(2 \pi)^{3} .
$$

Substitution of Eq. (6) into Eq. (5) gives the integral equation

$$
\begin{aligned}
\left(q^{2}+k^{2}\right) \mathbf{A}(\mathbf{q}, k) & -2 \gamma \int \frac{d p}{2 \pi} p \sin [(p-k) d / 2] \mathbf{A}(\mathbf{q}, p)= \\
& =-2 i \frac{\gamma}{d} \sin \frac{k d}{2} \mathbf{S}(\mathbf{q}) .
\end{aligned}
$$

Its solution is 


$$
\mathbf{A}(q, k)=-\frac{2 i \gamma}{d} \frac{\sin k d / 2}{q^{2}+k^{2}} \frac{\mathbf{S}(\mathbf{q})}{1+(\gamma / 2) \exp \{-q d\}} .
$$

Before finding the potential $\mathbf{A}(\rho, z)$ from Eq. (8), it is useful to estimate $\gamma$ numerically. It is $\gamma \approx$ $\approx 2 \pi \cdot 10^{-5}$ for the electron density $n \simeq 10^{12} \mathrm{~cm}^{-2}$, the insulator layer thickness $d \approx 10^{-6} \mathrm{~cm}$, and the effective mass of the pair $m \simeq 0.1 m_{0}$, where $m_{0}$ is the free electron mass. The $\gamma$ value is even lower for smaller $n$ and larger $m$. The condition $\gamma<<1$ is therefore oversatisfied for the experimental conditions. Equation (8) can therefore be restricted to the terms of the order of $\gamma$. Substituting the $\mathbf{A}(\mathbf{q}, k)$, calculated with this accuracy from Eq. (8), into Eq. (6), we obtain

$$
\begin{gathered}
A_{\theta}=\frac{2 \pi e \hbar n}{m \varphi p}\left[\sqrt{\rho^{2}+(z-d / 2)^{2}}-\right. \\
\left.-|z-d / 2|-\sqrt{\rho^{2}+(z+d / 2)^{2}}+|z+d / 2|\right] .
\end{gathered}
$$

We can now easily find the magnetic field excited by the planar vortex. The projection of the mag- netic field normal to the conducting layers is $H_{z}=\rho^{-1} \partial\left(\rho A_{\theta}\right) / \partial \rho$, i.e.,

$$
\begin{gathered}
H_{z}=2 \pi \frac{e \hbar n_{s}}{m c} \times \\
\left.\times\left\{\left[\rho^{2}+(z-d / 2)^{2}\right)\right]^{-1 / 2}-\left[\rho^{2}+(z+d / 2)^{2}\right]^{-1 / 2}\right\} .
\end{gathered}
$$

It is evident that $H_{z}$ is an odd function of $z$. The maximum value of $H_{z}$ is attained in the conducting layers, i.e., at $z= \pm d / 2$. The magnetic flux through the $z=d / 2$ plane, which is related to the electronhole vortex, is

$$
\Phi \equiv \int H_{z} \rho d \rho d \theta=\frac{4 \pi^{2} e \hbar n_{s} d}{m c} \equiv \gamma \Phi_{0} .
$$

The flux is not universal and depends on the superfluid density $n_{s}$ and the insulator thickness $d$. It constitutes only a small part of the flux quantum, $\Phi_{0} \equiv h c / 2 e$, produced by a vortex in ordinary superconductors.

The magnetic field component $H_{\rho}=-\partial A_{\theta} / \partial z$ is

$$
H_{\rho}=2 \pi \frac{e \hbar n_{s}}{m c \rho}\left[\operatorname{sign}\left(z-\frac{d}{2}\right)-\frac{z-d / 2}{\left[\rho^{2}+(z-d / 2)^{2}\right]^{1 / 2}}-\operatorname{sign}\left(z+\frac{d}{2}\right)+\frac{z+d / 2}{\left[\rho^{2}+(z+d / 2)^{2}\right]^{1 / 2}}\right] .
$$

Far from the vortex center or, more exactly, at $\rho>>d$, the field $H_{\rho}$ between the conducting layers decreases as $1 / \rho$. As a result, the $H_{\rho}$ contribution to the superfluid velocity $\mathbf{v}_{s}$ in Eq. (1) and the term proportional to $\nabla \varphi$ decrease as $1 / \rho$. The $1 / \rho$ decrease in the velocity $\mathbf{v}_{s}$ implies that the energy $U_{v}$ of the interaction between the vortices of opposite circulations is proportional to $\ln \left|\rho_{1}-\rho_{2}\right|$, where $\rho_{1}$ and $\rho_{2}$ are the vortex coordinates. We can show that the renormalization of the proportionality coefficient in $U_{v}$ due to the spatial electron-hole separation is about $\gamma$. But the energy $U_{v}$ determines the $T_{c}$ at which the pairs of vortices of opposite circulation dissociate, i.e., the temperature of the superfluid Berezinskii-Kosterlitz-Thouless transition. For this reason, the renormalization of $T_{c}$ is of the same order of magnitude. The spatial electron-hole separation therefore has virtually no effect on the temperature of the superfluid transition of the electron-hole pairs.

It is very important that because of the spatial electron-hole separation, the pairs which are as a whole electroneutral interact with the external magnetic field. As a result, the external field can lead to the vortex formation. The appearance of vortices in the external magnetic field $H_{0}$ is controlled by the energy

$E=\int d^{2} \rho\left[\frac{n_{s}}{2 m}\left(\hbar \nabla \varphi+\frac{e}{c} \hat{d \mathbf{z}} \times \mathbf{H}\right)^{2}+\left(\frac{H^{2}}{8 \pi}-\frac{\mathbf{H} \cdot \mathbf{H}_{0}}{4 \pi}\right) d\right]$.

The local field $\mathbf{H}$ consists of the external field $\mathbf{H}_{0}$ and the field created by the moving electron-hole pairs. In Eq. (13) we take into account that the main part of the energy of the field created by the pairs is concentrated in the space between the conducting layers. In this region the field $\mathbf{H}$ may be assumed equal to $\mathbf{H}_{0}+4 \pi e n_{s} \hat{\mathbf{z}} \times \mathbf{v}_{s} / c$ [cf. Eqs. (10) and (12)]. Using this expression and discarding the corrections on the order of $\gamma^{2}$, we obtain the following expression from Eq. (13):

$$
E=\int d^{2} \rho\left[\frac{\hbar^{2} n_{s}}{2 m}(\nabla \varphi)^{2}+\frac{\hbar n_{s} e d}{m c} \nabla \varphi\left(\hat{\mathbf{z}} \times \mathbf{H}_{0}\right)\right] .
$$




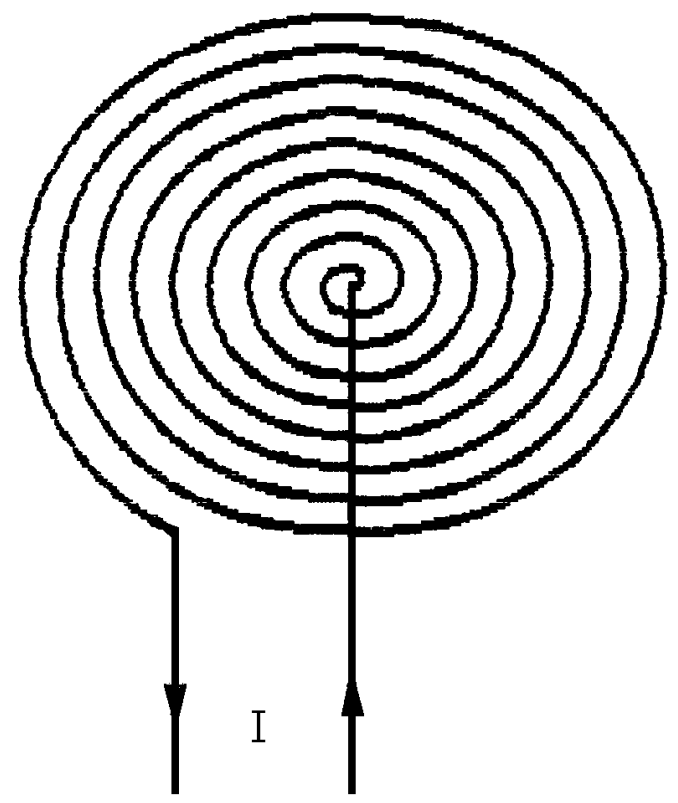

Fig. 1. Schematic diagram with two-dimensional azimuthal current

It can be concluded that with an appropriate strength and direction of the field $\mathbf{H}_{0}$, the second term can compensate for the vortex-related energy loss, $\int\left(\hbar^{2} n_{s} / 2 m\right)(\nabla \varphi)^{2} d^{2} \rho$. The vortex generation in the system thus becomes energy advantageous, as in the case of type-II superconductors at $H_{0}>H_{c 1}$.

The magnetic field excited by the two-dimensional current that circulates around the origin of coordinates is perhaps the most favorable field for generation of vortices. A schematic representation of this field is shown in Fig.1. If the two-dimensional density of the azimuthal current is $I$, then the magnetic field which is generated by the current at some distance from the edge of the structure is

$H_{0 \rho}=\frac{2 \pi I}{c}\left[\left(1+\frac{(z-l)^{2}}{\rho^{2}}\right)^{1 / 2}-\frac{|z-l|}{\rho}\right] \operatorname{sign}(z-l)$.

Here $l$ is the distance to the plane with the current $I$ reckoned from the midpoint of the insulator layer in the electron-hole structure.

Substituting the phase

$$
\varphi=\sum_{i} \arctan \left[\left(y-y_{i}\right) /\left(x-x_{i}\right)\right]
$$

$\left(x_{i}, y_{i}\right.$ are the coordinates of the $i$ th vortex) into Eq. (14), we find the energy $E$ of the vortices in the field $H_{0}$. The energy $E$ depends essentially on the size and shape of the system as the velocity $v_{s}$ decreases slowly from the vortex center. If the condenser superconductor is a three-layer disk of radius $R$, whose center coincides with the center of the structure shown in Fig. 1, and if the distance $l$ between this structure and the disk is much shorter than $R$, then the vortex energy will be

$$
E=\sum_{i=1}^{N} E_{\vartheta}\left(\rho_{i}\right)+\frac{1}{2} \sum_{i \neq j}^{N} U\left(\rho_{i}, \rho_{j}\right) .
$$

Here $E_{v}$ is the energy of one vortex, and $U$ is the energy of interaction between the vortices:

$$
\begin{gathered}
E_{v}(\rho)=\frac{\pi \hbar^{2} n_{s}}{m}\left(\ln \frac{R^{2}-\rho^{2}}{R \xi}-\frac{R-\rho}{\lambda}\right), \\
U\left(\rho_{i}, \rho_{j}\right)= \\
=\frac{\pi \hbar^{2} n_{s}}{m} \ln \frac{R^{2}-2 \rho_{i} \rho_{j} \cos \left(\theta_{i}-\theta_{j}\right)+\rho_{i}^{2} \rho_{j}^{2} / R^{2}}{\rho_{i}^{2}-2 \rho_{i} \rho_{j} \cos \left(\theta_{i}-\theta_{j}\right)+\rho_{j}^{2}} .
\end{gathered}
$$

The first term in Eq. (17) is the intrinsic vortex energy in the absence of the external magnetic field and the second term is the vortex-field interaction energy. Here we have introduced the notation

$$
\lambda^{-1}=\frac{4 \pi I e d}{\hbar c^{2}}
$$

Equation (18) takes into account the direct interaction between the vortices and their interactions with the images whose presence turns the $v_{s}$ component normal to the disk bound into zero.

In Eq. (17) the term proportional to $\lambda^{-1}$ makes the vortex state advantageous for small $\lambda$ (large currents $I$ ). To find the current $I_{c 1}$ at which the vortex creation becomes energy advantageous, we should take the number of vortices $N=1$ and the vortex coordinate $\rho_{1}=0$. Setting $E_{v}=0$, we have

$$
\lambda_{c 1}^{-1} \equiv \frac{4 \pi I_{c 1} e d}{\hbar c^{2}}=\frac{1}{R} \ln \frac{R}{\xi} .
$$

Substituting $d=10^{-6} \mathrm{~cm}$ into Eq. (20) and taking into account that $H_{0 \rho}=2 \pi I / c$ at $\rho>>d$, we find $H_{o \rho}^{c 1} \approx 1 \mathrm{Gs}$ for $R=1 \mathrm{~cm}$.

At $I>I_{c}$ the system has a finite number of vortices, which can be easily found for $I>>I_{c 1}$. In this case the vortices may be considered continuously distributed. Introducing the vortex density $n_{v}(\rho)$, we obtain

$$
\begin{gathered}
E=\int E_{v}(\rho) n_{v}(\rho) d^{2} \rho+ \\
+\frac{1}{2} \int n_{v}\left(\rho_{1}\right) U\left(\rho_{1}, \rho_{2}\right) n_{v}\left(\rho_{2}\right) d^{2} \rho_{1} d^{2} \rho_{2} .
\end{gathered}
$$


Varying Eq. (21) in $n_{v}(\rho)$ and setting the result equal to zero, we obtain the equation for the dependence of the vortex density on $\rho$. We can solve the equation exactly and thus show that at $\rho>>l$

$$
n_{v}(\rho)=(4 \pi \lambda \rho)^{-1} .
$$

The result is drastically different from that known for neutral superfluid systems and ordinary superconductors. In both cases the equilibrium vortex concentration (Onsager-Feynman and Abrikosov, respectively) is spatially uniform. We clearly see from Eq. (22) that $n_{v}$ in our case decreases with increasing $\rho$.

It is known that the Onsager-Feynman and Abrikosov vortices form a triangular lattice. The ques- tion then arises whether the vortices considered in this paper also form a lattice. Since $n_{v}(\rho)$, according to Eq. (22), decreases with increasing distance from the disk center, it is evident that the vortices cannot form a structure invariant under translations. Equation (22), however, admits a structure invariant against rotations. As such a structure we choose the structure where vortex cores are situated on the circumferences whose centers coincide with that of the disk. The number of vortices on the $i$ th circumference is $N_{i}$, and its radius is $\rho_{i}$. Performing in Eq. (16) summation over the vortex coordinates on the $i$ th circumference by the Poisson summation formula, we obtain the following expression for the vortex energy:

$$
\begin{aligned}
E=\sum_{i}\left\{E_{v}\left(\rho_{i}\right)+\frac{\pi \hbar^{2} n_{s}}{M}\left[2 \sum_{j(<i)} N_{j}\left(\ln \frac{R}{\rho_{i}}+\frac{1}{N_{i}} \ln \frac{1-\exp \left\{-N_{i} \ln R^{2} / \rho_{i} \rho_{j}\right\}}{1-\exp \left\{-N_{i} \ln \rho_{i} / \rho_{j}\right\}}\right)-\right.\right. \\
\left.\left.-\ln N_{i}+\left(N_{i}-1\right) \ln \frac{R}{\rho_{i}}+\ln \left(1-\exp \left\{-2 N_{i} \ln \frac{R}{\rho_{i}}\right\}\right)\right]\right\} N_{i} .
\end{aligned}
$$

This exact expression can be simplified considerably at $N_{i}>>1$. In the case the main contribution to the last sum in Eq. (23) is made by the terms for which $\left|\ln \rho_{j} / \rho_{i}\right|<<1$. For these terms $\left|\ln \rho_{j} / \rho_{i}\right| \simeq\left(\rho_{i+1}-\rho_{i}\right)|j-i| / \rho_{i}$. The terms with $\ln R^{2} / \rho_{i} \rho_{j}$ may be discarded far from the disk edge. Varying the resulting expression in $N_{i}$ and $\rho_{i}$ and setting the result equal to zero, we obtain a set of equations for these quantities. The solution for $R>>\rho_{i}>>\lambda$ is

$$
\begin{gathered}
N_{i}=\frac{\rho_{i+1}-\rho_{i}}{2 \lambda}, \\
\left(\rho_{i+1}-\rho_{i}\right)^{2}=4 \lambda \rho_{i} .
\end{gathered}
$$

Both results of Eq. (24) readily follow from simple physical considerations. The number of vortices on the $i$ th circumference is

$$
N_{i}=\int_{\rho_{i}}^{\rho_{i+1}} \int_{0}^{2 \pi} n_{v}(\rho) \rho d \rho d \theta .
$$

Substitution of $n_{v}(\rho)$ from Eq. (22) into this expression gives $N_{i}=\left(\rho_{i+1}-\rho_{i}\right) / 2 \lambda$. To obtain the other result of Eq. (24), we should take into account that the repulsion forces between the vortices of the same circulation are isotropic. It is therefore expected that the spatial distribution of vortices is locally isotropic. In this case the mean vortex spacing $2 \pi \rho_{i} / N_{i}$ for the particular circumference coincides with the mean vortex spacing $\rho_{i+1}-\rho_{i}$ on the neighboring circumferences. Substituting the value for $N_{i}$, we obtain $4 \pi \lambda \rho_{i} /\left(\rho_{i+1}-\rho_{i}\right)=\rho_{i+1}-\rho_{i}$, which coincides with Eq. (24) with an accuracy up to the multiplier $\pi$.

We have considered thoroughly the case of generation of planar vortices by the field of two-dimensional circular current. It is inferred that in an arbitrary magnetic field, within the limit where vortices are assumed continuously distributed, the vortex density is $n_{v}(\rho)=(e d / 2 \pi \hbar c)\left|\partial H_{0 z} / \partial z\right|$. In summary, a nonuniform magnetic field can, in general, excite vortices in the PSSEH systems. The nature of the vortex distribution in space needs to be studied.

The research described in this publication was made possible, in part, by Grant U2D200 from the Joint Fund of the Government of Ukraine and International Science Foundation. 
1. S. A. Moskalenko, Fiz. Tverd. Tela 4, 276 (1962); J. M. Blatt, K. W. Boer, and W. Brandt, Phys. Rev. 126, 1691 (1962).

2. L. V. Keldysh and Yu. V. Kopaev, Fiz. Tverd. Tela 6, 2791 (1964); B. I. Halperin and T. M. Rice, Solid State Phys. 21, 116 (1968).

3. R. R. Guseinov and L. V. Keldysh, Zh. Teor. Eksp. Fiz. 63, 2255 (1972) [Sov. Phys. JETP 36, 1193 (1972)].

4. S. I. Shevchenko, Fiz. Nizk. Temp. 2, 505 (1976) [Sov. J. Low Temp. Phys. 2, 251 (1976)].

5. Yu. E. Lozovik and V. I. Yudson, Zh. Eksp. Teor. Fiz. 71, 738 (1976) [Sov. Phys. JETP 44, 389 (1976)].

6. S. I. Shevchenko, Phys. Rev. Lett. 72, 3242 (1994).

7. J. E. Golub, K. Kash, J. P. Harbison, and L. T. Florez, Phys. Rev. B41, 8564 (1990).

8. U. Sivan, P. M. Solomon, and H. Shtrikman, Phys. Rev. Lett. 68, 1196 (1992).

9. G. D. Gilliland, A. Antonelli, D. J. Wolford, K. K. Bajaj, J. Klem, and J. A. Bradley, Phys. Rev. Lett. 71, 3717 (1993).

10. L. V. Butov, A. Zrenner, G. Abstreiter, G. Böhm, and G. Weiman, Phys. Rev. Lett. 73, 304 (1994).

11. J. P. Eisenstein, L. N. Pfeiffer, and K. W. West, Phys. Rev. Lett. 74, 1419 (1995).

12. L. Swierkowski, J. Szymanski, and Z. W. Gortel, Phys. Rev. Lett. 74, 3245 (1995).
13. X. Zhu, P. B. Littlewood, M. S. Hybertsen, and T. M. Rice, Phys. Rev. Lett. 74, 1633 (1995).

14. A. B. Dzyubenko and G. E. W. Bauer, Phys. Rev. B51, 14524 (1995).

15. G. Vignale and A. H. MacDonald, Phys. Rev. Lett. 76 , 2786 (1996).

16. Y. Naveh and B. Laikhtman Phys. Rev. Lett. 77, 900 (1996).

17. D. W. Snoke, J. P. Wolfe, and A. Mysyrowicz, Phys. Rev. B41, 11171 (1990); Jia Ling Lin and J. P. Wolfe, Phys. Rev. Lett. 71, 1222 (1993).

18. Y. Kuramoto and C. Horie, Solid State Commun. 25, 713 (1978).

19. I. V. Lerner and Yu. E. Lozovik, Zh. Eksp. Teor. Fiz. 78, 1167 (1980) [Sov. Phys. JETP 51, 588 (1980)]; I. V. Lerner and Yu. E. Lozovik, Zh. Eksp. Teor. Fiz. 80, 1488 (1981) [Sov. Phys. JETP 53, 763 (1981)]; A. B. Dzyubenko and Yu. E. Lozovik, Fiz. Tverd. Tela (Leningrad) 26, 1540 (1984).

20. D. Yoshioka and A. H. MacDonald, J. Phys. Soc. Jpn. 59, 4211, (1990).

21. X. M. Chen and J. J. Quinn, Phys. Rev. Lett. 67, 895 (1991). 